\title{
Theory for normal state critical Kapitza resistance of ${ }^{4} \mathrm{He}$
}

\author{
R. A. Ferrell \\ Center for Theoretical Physics of the Department of Physics and Astronomy, University of Maryland, \\ College Park, Maryland 20742, USA \\ E-mail: rferrell@muppetn.umd.edu \\ J. K. Bhattacharjee \\ Indian Association for the Cultivation of Science, Jadavpur, Calcutta 700 032, India \\ S. I. Mukhin \\ Moscow State Institute for Steel and Alloys, Theoretical Physics Dept., \\ Leninskii prospect 4, 117936 Moscow, Russia \\ E-mail: sergeimoskow@glasnet.ru
}

\begin{abstract}
We study a steady state, bearing a heat current, $Q_{0}$, in the normal state of liquid ${ }^{4} \mathrm{He}$ with constraining geometry, near the lambda point, at temperatures $T$ greater than the lambda temperature, $T_{\lambda}$. Critical order parameter fluctuations near the boundary are incorporated in the expression for the non-local thermal resistivity. The Kapitza resistance is manifested by the additional temperature rise at the boundary, as compared with the bulk-extrapolated value. Sensitivity of the calculations to the application of the Dirichlet boundary condition is discussed.
\end{abstract}

PACS: $64.60 . \mathrm{Ht}, 67.40 . \mathrm{Hf}, 67.40 . \mathrm{Pm}$

In the region of the lambda point of ${ }^{4} \mathrm{He}$ the critical Kapitza resistance is a strong function of temperature. This behavior is predicted by theory and has been measured experimentally. It is puzzling that the recent experimental data [1] and the theoretical results [2] for the additional boundary contribution to the thermal conductivity of ${ }^{4} \mathrm{He}$ just above the $\lambda$-point show a significant discrepancy. Understanding the physical source of this discrepancy is important for the general theory of critical fluctuations at a second-order phase transition. In this paper we calculate the Kapitza resistance in a different way from that of Frank and Dohm [2] by modeling a boundary with the heat source imbedded in the liquid helium in the plane $z=0$. Such a source produces the outward flow

$$
Q(z)=Q_{0} \operatorname{sign} z=\left\{\begin{array}{c}
Q_{0}, z>0, \\
-Q_{0}, z<0 .
\end{array}\right.
$$

The resulting temperature distribution would give the gradient

(C) R. A. Ferrell, J. K. Bhattacharjee, and S. I. Mukhin, 1998

$$
\frac{d T_{\infty}}{d z} \equiv T_{\infty}^{\prime}(z)=-\rho(0, \kappa) Q(z),
$$

in the absence of any boundary effect, with $\rho(0, \kappa)=\lambda^{-1}(0, \kappa)$ the thermal resistivity, i.e., the reciprocal of the thermal conductivity. Then, the Kapitza resistance is manifested by the difference:

$$
\Theta(z)=T(z)-T_{\infty}(z) .
$$

The choice of zero in temperature is irrelevant and does not affect this difference. The wave number dependent non-local resistivity, $\rho(k, \kappa)$, determines the spatial derivative, $\Theta^{\prime}(z)$, by the operator equation

$$
\Theta^{\prime}(z)=T^{\prime}(z)-T_{\infty}^{\prime}(z)=-[\hat{\rho}-\rho(0, \kappa)] Q(z) .
$$

In the Fourier representation this becomes

$$
i k \tilde{\Theta}(k)=-[\rho(k, \kappa)-\rho(0, \kappa)] \tilde{Q}(k) .
$$

Here we use the following notations: 


$$
\begin{gathered}
\tilde{Q}(k) \equiv \int_{-\infty}^{\infty} d z \exp (-i k z) Q(z)=-\frac{2 i}{k} Q_{0}, \\
\tilde{\Theta}(k) \equiv \int_{-\infty}^{\infty} d z \exp (-i k z) \Theta(z)
\end{gathered}
$$

Combining Eqs. (5) and (6) we readily get

$$
\tilde{\Theta}(k)=\frac{2}{k^{2}}[\rho(k, \kappa)-\rho(0, \kappa)] Q_{0} .
$$

The Kapitza resistance is consequently

$$
R_{K}=\frac{\Theta(0)}{Q_{0}}=\frac{2}{\pi} \int_{0}^{\infty} \frac{d k}{k^{2}}[\rho(k, \kappa)-\rho(0, \kappa)] .
$$

Making use of the bulk thermal conductivity,

$$
\lambda=\rho(0, \kappa)^{-1} \sim \kappa^{-x / \nu},
$$

we can approximate the non-local resistivity by

$$
\rho(k, \kappa)=\lambda^{-1}\left[1+(k / a \kappa)^{2}\right]^{x / 2 v}
$$

with $a=O(1)$. The thermal conductivity critical exponent in the crossover region is $x$ and the critical exponent of the correlation length is $\nu, \xi=\kappa^{-1}$.

Thus,

$$
R_{K}=\frac{a \xi}{\lambda} I
$$

where the definite integral is

$$
I=\frac{2}{\pi} \int_{0}^{\infty} \frac{d u}{u^{2}}\left[\left(1+u^{2}\right)^{x / 2 v}-1\right] .
$$

This integral converges at both upper and lower limits due to $x / v<1$. Integration by parts yields

$$
I=\frac{2}{\pi} \frac{x}{v} \int_{0}^{\infty} \frac{d u}{\left(1+u^{2}\right)^{1-x / 2 v}} .
$$

Noting that $1-x / 2 v>1 / 2$, and passing to the new variable $\left(u=\tan \chi, d u=\sec ^{2} \chi\right)$, the integral reduces to the Euler beta-function, $\beta(m, n)$ :

$$
I=\frac{2}{\pi} \beta\left(\frac{1}{2}, \frac{1}{2}-\eta\right) ; \eta \equiv \frac{x}{2 v} .
$$

It is convenient to rewrite Eq. (11) in the form

$$
R_{K}=\frac{l_{\text {eff }}}{\lambda}
$$

with the amplitude of the effective thickness of the critical boundary layer

$$
l_{\text {eff }}=A \xi
$$

given by

$$
A=a I .
$$

From the slope of the log-log plot of Ahlers' data [3] we obtain

$$
x=0.46 .
$$

This crossover effective exponent is well accounted for by «quasi-scaling» theory [5], in which the asymptotic dynamic scaling exponent of 0.40 is increased by the factor of $1+z_{Q}$, where $z_{Q}$ was found to equal 0.18 . This brought the theoretically expected quasi-scaling exponent up to

$$
x_{Q}=0.40 \cdot 1.18=0.47 \text {, }
$$

in good accord with the experimental value of $x$. Substituting this value of $x$ into Eq. (14), with $v=0.672$, we obtain $\eta=0.343$, which yields $\beta(1 / 2,1 / 2-\eta)=7.66$ and $I=1.673$. From the «rule of thumb» [6] and the two-term $\varepsilon$ expansion [7] we have $a=2$, so that $A=2.35$.

In order to compare this result with the theory of Frank and Dohm [2] we have deduced their value for this coefficient by reading off the numerical values for $R_{K}$ from their Fig. 3 in Ref. 2. It suffices to consider the case $t=10^{-6}$, for which they evidently predicted the value $R_{K}=0.0268 \mathrm{~K} \cdot \mathrm{cm}^{2} / W$. Multiplying by Ahlers' [3,4] thermal conductivity at the saturated vapor pressure and at this same temperature, namely, $\lambda=6.24 \cdot 10^{-3} \mathrm{~W} / \mathrm{K} \cdot \mathrm{cm}$ we find $l_{\text {eff }}^{F D}=1.67 \cdot 10^{-4} \mathrm{~cm}$. Dividing by the correlation length

$$
\xi=\xi_{0} t^{-v}=1.40 \cdot 10^{-8} \cdot 10^{6 v} \mathrm{~cm}=1.49 \cdot 10^{-4} \mathrm{~cm},
$$

we obtain for the Frank-Dohm theory the coefficient $A_{F D}=1.12$.

In comparing data [1] with the Frank-Dohm theory [2], Lipa and Li noted a significant discrepancy in magnitude. The size of the boundary effect that they observed was evidently approximately five times greater than that predicted by Frank and Dohm. Our treatment, presented above, predicts a stronger effect than that of Frank and Dohm by the factor of 2.1 (from A and $l_{\text {eff }}^{F D}$ ). This factor can be expected to get bigger when we improve our idealized «embedding» theory by introducing the Di- 
richlet boundary condition at the heat source. The increase, however, is unlikely to be large enough to account fully for the observed magnitude of the effect.

The work has been supported by NASA Grant NAG3-1867.

1. J. A. Lipa, Adv. Space Res. 13, 61 (1993); J. A. Lipa and Q. Li, Czech. J. Phys. 46, Suppl. S1 (1996).

2. D. Frank and V. Dohm, Z. Phys. B84, 443 (1991).
3. G. Ahlers, in: Proc. of the XII'th Int. Conf. on Low Temperature Physics, Kyoto, 1970, E. Kanda (ed.), Keigaku, Tokyo (1971), p. 21.

4. G. Ahlers, in: Physics of Liquid and Solid Helium, K. H. Benneman and J. B. Ketterson (eds.), Wesley, New York (1976), vol. 1, Fig. 36.

5. R. A. Ferrell and J. K. Bhattacharjee, Phys. Rev. B28, 121 (1983).

6. R. A. Ferrell, J. Phys. (Paris) 32, 85 (1971).

7. R. A. Ferrell and J. K. Bhattacharjee, J. Low Temp. Phys. 36, 166 (1979). 